\title{
Safety and feasibility of extending interval flushing every 3 months for maintenance of chemoport in colorectal cancer patients after completion of curative intended treatments
}

\author{
Sang-Bo Oh, Kwonoh Park, Jae-Joon Kim, So Yeon Oh \\ Medical Oncology and Hematology, Department of Internal Medicine, Pusan National University Yangsan Hospital, \\ Pusan National University School of Medicine, Yangsan, Korea.
}

Background: Colorectal cancer (CRC) patients treated with curative intended surgery undergo 5-FU continuous infusion based chemotherapy using chemoport $(\mathrm{CP})$ in case of high risk of recurrence. Approximately $30 \%$ patients relapse after the completion of therapy, especially within 2 years. Hence, many of high risk CRC patients keep the CP during 6-24 months after treatment, with regular intervals of $\mathrm{CP}$ flushing. The manufacturer recommends monthly flushing for maintain $\mathrm{CP}$, however the time interval lacks scientific evidence. Considering that surveillance visiting after treatment completion is usually scheduled every 3-6 months, monthly flushing is excessive and inconvenient. We have applied the 3-month interval flushing since 2010. The aim of this study is to investigate whether the extended maintenance intervals is safe and feasible.

Methods: A retrospective cohort was conducted in CRC patients who underwent curative intended surgery and perioperative chemotherapy using CP between 2010 and 2017. Patient were enrolled if CP was maintained at least 6 months with 3-month interval flushing using heparin, while patient who were recurred within 1 month, removed CP within 6 months without definitive causes, or violation of flushing interval (30 days window was permitted) were excluded. The primary end points were the functional CP maintenance rate, including maintenance of $\mathrm{CP}$ at least 6 months, planned CP removal after 6 months, and regaining the use of $\mathrm{CP}$ at the time of recurrence.

Table 1. Baseline patient's characteristic

\begin{tabular}{lcc}
\hline Characteristic & Total (N= 154) & $\%$ \\
\hline Age, years, median (range) & $62(36-76)$ & \\
Sex & & $50.6 \%$ \\
Male & 78 & $49.4 \%$ \\
Female & 76 & \\
Primary cancer site & & $3.2 \%$ \\
Cecal cancer & 5 & $26.6 \%$ \\
Ascending & 41 & $5.8 \%$ \\
Transverse & 9 & $5.2 \%$ \\
Descending & 8 & $40.3 \%$ \\
Sigmoid & 62 & $3.9 \%$ \\
Rectosigmoid & 6 & $14.9 \%$ \\
Rectum & 23 & \\
Stage & & $5.2 \%$ \\
II & 83 & $24.7 \%$ \\
III & 38 & $53.9 \%$ \\
IV - synchronous & 25 & $16.2 \%$ \\
IV - metachronous & & \\
\hline
\end{tabular}

Results: A total of 214 CRC patients underwent curative intended treatments during the study period. Among them, 6 patients (early recurrence within 1 month) and 54 patients (violation of flushing interval) were excluded, finally 154 patients were analyzed. Disease stages were stage II (38, 25\%), III (83, $54 \%)$, IV-synchronous $(25,16 \%)$, and IV-metachronous $(8,5 \%)$. Mean flushing interval was 98.4 days $(95 \% \mathrm{Cl}, 96.2$ - 100.6; range, 60-120). At the Dec 2018, 35 patients kept the CP, 92 were planned removal, and 24 were reused the $\mathrm{CP}$, while two patients had to unexpectedly remove due to CP site infection and pain. Thus, the functional CP maintenance rate was $98.7 \%$ $(152 / 154)$. Thirty eight patients were relapsed and 30 were treated with intravenous chemotherapy. Among them, 26 patients $(87 \%)$ were reused the existing CP without re-insertion procedure.

Conclusions: Our study demonstrated that 3-month interval flushing is safe and feasible in CRC patients. Extended time interval up to 3 months might be considered because it is compatible with $\mathrm{CRC}$ surveillance visit schedules and convenient for patients

Table 2. Patients' heparin flushing status

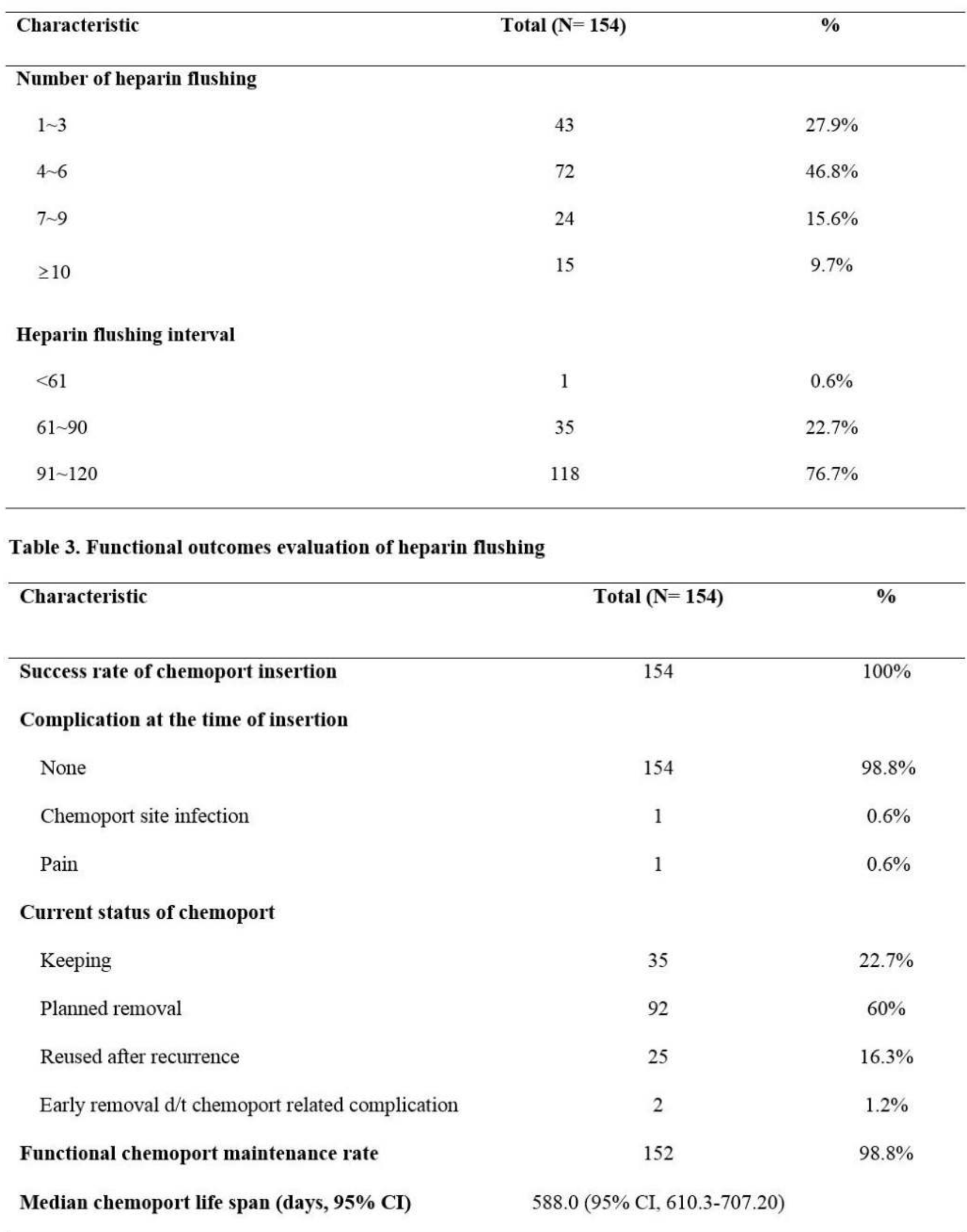

